\title{
¿Qué estrategias de intervención funcionan en la educación de los niños con trastorno del espectro autista? Revisión de evidencias en la literatura científica
}

\author{
Raúl Tárraga-Mínguez (Universitat de València) \\ Pilar Sanz-Cervera (Universitat de València)
}

Recepción: 17.09.2018 | Aceptado: 30.09.2018

Correspondencia a través de ORCID: Raúl Tárraga

iD 0000-0002-4458-5763

Citar: Tárraga-Mínguez, R. y Sanz-Cervera, P. (2018). ¿Qué estrategias de intervención funcionan en la educación de los niños con trastorno del espectro autista? Revisión de evidencias en la literatura científica. ReiDoCrea, 7, 279-287.

\begin{abstract}
Resumen: La creciente escolarización de estudiantes con trastorno del espectro autista (TEA) en entornos inclusivos requiere que los profesionales, ya no solo especialistas en el ámbito de la educación especial, sino también los maestros generalistas, conozcan procedimientos de intervención empíricamente efectivos para poder trabajar con este tipo de alumnado. Para ello, en el presenta trabajo se incluye una revisión de estudios de metaanálisis o de revisión cualitativa, que han analizado diferentes tipos de intervención educativa con estudiantes con TEA. Las conclusiones obtenidas indican que es necesario que los resultados sean robustos en diferentes estudios y que cumplan con los estándares propuestos por el Council of Exceptional Children. En concreto, se ha obtenido que las intervenciones basadas en análisis conductual aplicado parecen cumplir con estos estándares, además de ofrecer resultados positivos. También se considera el papel de las familias como agentes de intervención imprescindibles para poder transferir los resultados a la vida diaria de los niños con TEA. Esta revisión de la literatura pretende proporcionar algunas certezas que contribuyan a mejorar la práctica docente de los profesionales que intervienen con niños con TEA, además de incluir algunas cuestiones que invitan a la reflexión sobre la eficacia de las diferentes intervenciones.
\end{abstract}

Palabras clave: Autismo | Intervención Educativa

What intervention strategies work in the education of children with autism spectrum disorder? A systematic review in the scientific literature

\begin{abstract}
The increasing schooling of students with autism spectrum disorder (ASD) in inclusive settings requires that professionals, not only specialists in the field of special education, but also regular teachers, know empirically effective intervention procedures to be able to work with this type of students. To do this, this paper presents a review of meta-analysis studies or qualitative reviews, which have analyzed different types of educational interventions with students with ASD. The conclusions obtained indicate that it is necessary that the results will be robust in different studies and that they meet with the standards proposed by The Council of Exceptional Children. In particular, it has been obtained that interventions based on applied behavioral analysis seem to meet with these standards, in addition to offering positive results. The role of families as essential intervention agents to transfer the results to the daily life of children with ASD is also considered. This literature review aims to provide some certainties that contribute to improving the teaching experience of professionals involved with children with ASD, in addition to including some issues that invite reflection on the effectiveness of the different interventions.
\end{abstract}

Keywords: Autism | Educational Treatment

\section{Introducción}

La escolarización de estudiantes con trastorno del espectro autista (TEA) supone actualmente un importante desafío para los profesionales del ámbito de la educación. Los responsables de las tutorías, profesorado especialista de asignaturas específicas, profesionales de la orientación y educadores/as de educación especial, son los agentes encargados de que verdaderamente se puedan cumplir los objetivos educativos que se plantean con estos estudiantes, y de que se materialicen verdaderamente en la práctica las metas de la educación inclusiva. Sin embargo, esta 
responsabilidad no es sencilla de llevar a la práctica, sino que supone un importante reto del que depende la calidad de la educación de los niños con TEA.

Los motivos por los que la educación de estos estudiantes supone un desafío, son (entre otros), los siguientes:

En primer lugar, la prevalencia del TEA ha aumentado de una manera significativa en los últimos años en los estudios internacionales (Russell, Collishaw, Golding, Kelly y Ford, 2015), hasta situarse en nuestro país aproximadamente en el $0.61 \%$ (Fortea, Escandell y Castro, 2013). El aumento de estas cifras no implica necesariamente que ahora haya más estudiantes con TEA que en el pasado. Las revisiones sobre el tema nos sugieren que factores como: el mayor conocimiento del diagnóstico por parte de los profesionales que deben identificarlo, la disponibilidad de más y mejores herramientas diagnósticas y los cambios que se han introducido en los criterios diagnósticos, son probablemente algunas de las causas del aumento de las cifras de prevalencia.

Reconocer mejor el TEA y visibilizarlo es un aspecto positivo que mejora la situación anterior, pero este reconocimiento debe ir acompañado posteriormente de las medidas educativas adecuadas.

Precisamente, la ausencia de una formación específica para atender a las necesidades educativas de los niños con TEA, es otro de los desafíos a los que nos encontramos. Los estudios que han tratado de valorar el grado de conocimiento y formación de los profesionales de la educación, sugieren que todavía existe desconocimiento por parte del profesorado sobre el TEA, y lo que probablemente es peor, existen algunas falsas creencias que pueden ir en detrimento de la calidad de la educación de estos estudiantes (Sanz-Cervera, Fernández-Andrés, Pastor-Cerezuela y Tárraga-Mínguez, 2017). Esta falta de formación no es homogénea entre todos los profesionales de la educación. El profesorado especialista en educación especial posee más conocimientos sobre el ámbito que el profesorado generalista (SanzCervera et al., 2017).

Esta situación, es lógica si atendemos a la formación inicial que recibe el profesorado generalista y el especialista, y responde a un planteamiento de la formación del profesorado en nuestro país en la que la educación especial tiene en la práctica figuras profesionales específicas, cuya presencia contribuye a proporcionar una respuesta educativa desde su ámbito de especialización (Vélez-Calvo, TárragaMínguez, Fernández-Andrés y Sanz-Cervera, 2016). Sin embargo, desgraciadamente, encontramos situaciones en las que la presencia de estos profesionales especialistas con conocimientos sobre el TEA, y la falta de conocimiento del profesorado generalista, se acaba convirtiendo en una circunstancia que no beneficia las aspiraciones de la educación inclusiva, ya que favorece situaciones en que las intervenciones se realizan fuera del contexto ordinario.

A este respecto, otro de los retos a los que debemos hacer frente en la educación de un niño con TEA, es la coordinación entre profesionales. Habitualmente, un mismo niño con TEA recibe atención educativa por parte de su tutor/a y del profesorado especialista, como el resto de estudiantes. Pero, además, pueden intervenir: el maestro/a de pedagogía terapéutica, el maestro/a de audición y lenguaje, el educador/a de educación especial, el orientador/a (aunque habitualmente hace una intervención indirecta), y posibles profesionales externos al centro (personal de asociaciones, logopedas, psicólogos/as especialistas, etc.). Este elevado número de profesionales complica la coordinación, lo que es especialmente relevante en el caso de niños con TEA, que experimentan más dificultades de lo habitual cuando se enfrentan a cambios repentinos en sus dinámicas diarias. 
Finalmente, las propias características del TEA, hacen que se trate de un diagnóstico especialmente complicado para muchos profesionales de la educación: las dificultades en la comunicación e interacción social que experimentan los niños con TEA les impiden en muchas ocasiones interpretar las situaciones cotidianas del aula y comunicar de manera adecuada sus intereses y sentimientos. Ello puede generar problemas de conducta que resultan complicados de gestionar para el profesorado. Por otro lado, algunas características intrínsecas al TEA, como la presencia de patrones restrictivos de comportamientos e intereses, pueden dificultar las relaciones de estos niños con el resto de compañeros, lo que también genera dificultades en la adaptación escolar (Humphrey y Hebron, 2015). Incluso, algunas conductas intrínsecas al diagnóstico, como las estereotipias o la ecolalia, pueden ser interpretadas por el profesorado como problemas de conducta, cuando en realidad son únicamente manifestaciones conductuales del diagnóstico.

Ante estos retos, es necesario que los profesionales de la educación que desarrollan su trabajo a diario en el aula con niños con TEA, dispongan de los conocimientos adecuados acerca de qué procedimientos de intervención son verdaderamente efectivos para alcanzar los objetivos que se proponen, y que además hayan demostrado su efectividad de manera consistente en diferentes estudios.

Ante esta necesidad de conocer qué procedimientos de intervención se han demostrado como empíricamente efectivos, el objetivo del presente trabajo es realizar una revisión de los estudios de metaanálisis y estado del arte que han analizado diferentes aproximaciones de intervención educativa con estudiantes con TEA. Consideramos que esta revisión de la literatura puede contribuir a mejorar la práctica docente de los profesionales encargados de la educación de niños con TEA, ya que proporciona información clara, expuesta de manera accesible sobre la efectividad de estas intervenciones.

\section{Método}

Para alcanzar el objetivo de esta revisión, se empleó la base de datos Psyclnfo, con la intención de localizar artículos de revisión de la literatura y metaanálisis sobre intervenciones mediante un procedimiento específico dirigido a estudiantes con TEA.

El motivo por el que se escogió revisar únicamente estos dos tipos de trabajos fue el de incluir en este artículo únicamente intervenciones que hayan mostrado su eficacia de una manera sistemática, con indicadores robustos de su eficacia, basados en investigaciones científicas. Se descartaron por tanto trabajos de intervención con un único sujeto o grupo de sujetos, ya que el interés estaba en localizar trabajos que revisaran diferentes intervenciones.

Se realizaron dos búsquedas. En la primera de ellas se emplearon las palabras clave: autism, intervention, y literature review; y se obtuvieron un total de 227 resultados. En la segunda búsqueda se sustituyó la palabra clave literature review por metaanalysis; y se obtuvieron un total de 71 resultados. Posteriormente, se eliminaron los resultados que: estaban duplicados en las dos búsquedas, no eran revisiones cualitativas de la literatura o metaanálisis, no estaban centrados específicamente en estudiantes con TEA, trabajaban algún aspecto no directamente relacionado con el ámbito educativo, que requerían de la intervención de personal sanitario especializado (como conducta alimentaria o problemas de salud graves), o eran publicaciones diferentes a artículos científicos. Tras aplicar estos filtros, la búsqueda se redujo a un total de 10 resultados, que son los que se resumen en la siguiente sección. 


\section{Resultados}

\begin{tabular}{|c|c|c|c|}
\hline Autor/es (año) & $\mathbf{N}$ & Intervención & Resultados \\
\hline Gerow et al. (2018) & $\begin{array}{l}\text { Revisión cualitativa de } 26 \text { estudios. } \\
\text { Total participantes: } 78 \text { padres y madres de } \\
69 \text { niños con TEA. } \\
\text { Edad: } 3-14 \text { años (aunque la mayoría se } \\
\text { concentraba en el rango de } 3-5 \text { años). }\end{array}$ & $\begin{array}{l}\text { Entrenamiento en comunicación funcional } \\
\text { implementado por padres. }\end{array}$ & $\begin{array}{l}\text { La revisión sugiere que las intervenciones son eficaces para reducir el } \\
\text { comportamiento desafiante de los niños con TEA. } \\
\text { Sin embargo, pocos estudios ofrecen información sobre el grado de fidelidad en la } \\
\text { implementación de las intervenciones. } \\
\text { Se concluye que es necesaria más investigación para poder afirmar que este tipo } \\
\text { de intervenciones son eficaces. }\end{array}$ \\
\hline Hillman (2018) & $\begin{array}{l}\text { Revisión cualitativa de } 4 \text { estudios. } \\
\text { Total participantes: } 8 \text { niños con TEA. } \\
\text { Edad: } 4-11 \text { años. }\end{array}$ & Terapia de juego basada en el niño. & $\begin{array}{l}\text { Los resultados de las intervenciones apuntan resultados positivos en } \\
\text { sintomatología TEA. Sin embargo, es necesario un volumen mucho mayor de } \\
\text { investigación para confirmar con evidencias la efectividad de esta aproximación de } \\
\text { intervención. }\end{array}$ \\
\hline $\begin{array}{l}\text { Makrygianni, Gena, Katoudi } \\
\text { y Galanis (2018) }\end{array}$ & $\begin{array}{l}\text { Metaanálisis de } 29 \text { estudios. } \\
\text { Total participantes: } 831 \text { niños con TEA. } \\
\text { Edad: } 25.10 \text { - } 66.31 \text { meses. }\end{array}$ & $\begin{array}{l}\text { Intervenciones basadas en análisis } \\
\text { conductual aplicado. }\end{array}$ & $\begin{array}{l}\text { Los resultados de los estudios incluidos en el metaanálisis produjeron diferente } \\
\text { grado de mejora en las diferentes áreas. } \\
\text { Las intervenciones fueron muy efectivas en la mejora de habilidades cognitivas; } \\
\text { entre moderadas y muy efectivas en habilidades de comunicación, habilidades de } \\
\text { lenguaje expresivo y receptivo; moderadamente efectivas en la mejora de las } \\
\text { puntuaciones de Cl no verbal, conducta adaptativa y socialización; y tuvieron } \\
\text { incidencia baja en mejora de habilidades de la vida diaria. }\end{array}$ \\
\hline $\begin{array}{l}\text { McGill, Baker y Busse } \\
(2014)\end{array}$ & $\begin{array}{l}\text { Metaanálisis de } 77 \text { estudios de caso. } \\
\text { Total participantes: } 77 \text { niños con TEA. } \\
\text { Edad: 2-15 años. }\end{array}$ & $\begin{array}{l}\text { Intervenciones basadas en historias } \\
\text { sociales para reducir conductas } \\
\text { desafiantes. }\end{array}$ & $\begin{array}{l}\text { Las historias sociales tienen diferente grado de efectividad en la reducción de } \\
\text { conductas desafiantes en función de algunas características moduladoras. Las } \\
\text { intervenciones más efectivas fueron las que se llevaron a cabo en contexto escolar } \\
\text { o por parte de los investigadores. También fueron más efectivas las intervenciones } \\
\text { que planteaban como conductas objetivo conductas verbales. }\end{array}$ \\
\hline $\begin{array}{l}\text { Murza, Schwartz, Hahs- } \\
\text { Vaughn y Nye (2016) }\end{array}$ & $\begin{array}{l}\text { Revisión cualitativa y metaanálisis de } 15 \\
\text { estudios. Total participantes: } 410 \text { niños con } \\
\text { TEA en grupos experimentales y } 284 \text { en } \\
\text { grupos de comparación. Edad: } 11-152 \\
\text { meses. }\end{array}$ & $\begin{array}{l}\text { Intervenciones orientadas a la mejora de la } \\
\text { atención compartida. }\end{array}$ & $\begin{array}{l}\text { Los resultados del metaanálisis proporcionan evidencias robustas de la eficacia de } \\
\text { las intervenciones orientadas a la mejora de la atención compartida en niños con } \\
\text { TEA. }\end{array}$ \\
\hline $\begin{array}{l}\text { Nevill, Lecavalier y Straton } \\
\text { (2018) }\end{array}$ & $\begin{array}{l}\text { Metaanálisis de } 19 \text { estudios. } \\
\text { Total participantes: } 608 \text { niños en grupos } \\
\text { experimentales y } 597 \text { en grupos de }\end{array}$ & $\begin{array}{l}\text { Intervenciones mediadas por padres } \\
\text { basadas en dos modelos: comunicación } \\
\text { social o mejora de conducta en entornos }\end{array}$ & $\begin{array}{l}\text { El resultado del metanálisis indicó que la intervención mediada por los padres } \\
\text { produjo únicamente pequeñas mejorías en la reducción de la sintomatología TEA, } \\
\text { la socialización y la cognición. Las mejoras en comunicación y lenguaje fueron }\end{array}$ \\
\hline
\end{tabular}




\begin{tabular}{|c|c|c|c|}
\hline & $\begin{array}{l}\text { comparación. } \\
\text { Edad: } 15-72 \text { meses. }\end{array}$ & naturales. & muy reducidas. \\
\hline $\begin{array}{l}\text { Parsons, Cordier, Munro, } \\
\text { Joosten y Speyer (2017) }\end{array}$ & $\begin{array}{l}\text { Metaanálisis de } 20 \text { estudios. } \\
\text { Total participantes: } 925 \text { niños con TEA. } \\
\text { Edad: } 21 \text { meses - } 14 \text { años. }\end{array}$ & $\begin{array}{l}\text { Intervenciones orientadas a la mejora del } \\
\text { uso pragmático del lenguaje. }\end{array}$ & $\begin{array}{l}\text { El resultado del metaanálisis muestra que este tipo de intervenciones produce } \\
\text { resultados positivos durante la infancia (no en la adolescencia). La participación de } \\
\text { la familia en la intervención es un factor que aumenta las posibilidades de mejora. } \\
\text { Sin embargo, es necesario confirmar que las mejoras se generalizan a contextos } \\
\text { diferentes de la intervención. }\end{array}$ \\
\hline $\begin{array}{l}\text { Sanz-Cervera, Fernández- } \\
\text { Andrés, Pastor-Cerezuela y } \\
\text { Tárraga-Mínguez (2018) }\end{array}$ & $\begin{array}{l}\text { Revisión cualitativa de } 14 \text { estudios. } \\
\text { Total participantes: } 597 \text { niños con TEA. } \\
\text { Edad: 2-11 años (salvo un estudio con } \\
\text { adultos). }\end{array}$ & $\begin{array}{l}\text { Intervenciones basadas en la metodología } \\
\text { TEACCH. }\end{array}$ & $\begin{array}{l}\text { Todos los estudios mostraron mejoras en una reducción de sintomatología TEA y } \\
\text { conductas desadaptativas tras la intervención basada en metodología TEACCH. } \\
\text { Sin embargo, la investigación sobre esta metodología debe superar algunas } \\
\text { limitaciones, como el reducido número de estudios o la heterogeneidad de las } \\
\text { características de los participantes de las investigaciones. }\end{array}$ \\
\hline Tachibana et al. (2017) & $\begin{array}{l}\text { Revisión sistemática y metaanálisis de } 32 \\
\text { estudios. } \\
\text { Total participantes: } 594 \text { niños con TEA. } \\
\text { Edad: menores de } 6 \text { años. }\end{array}$ & $\begin{array}{l}\text { Intervenciones en niños en edad preescolar } \\
\text { divididas en tres grandes aproximaciones: } \\
\text { análisis conductual aplicado, intervención } \\
\text { orientada a la comunicación social y modelo } \\
\text { de intervención multimodal. }\end{array}$ & $\begin{array}{l}\text { Los resultados de las diferentes intervenciones produjeron mejoras en reciprocidad } \\
\text { e interacción social y coordinación educativa de los padres. } \\
\text { Sin embargo, es necesario realizar más investigación para asegurar que estas } \\
\text { mejoras se generalizan a otras intervenciones y no dependen de las } \\
\text { particularidades de cada estudio. } \\
\text { Los datos disponibles tampoco permiten diferenciar qué tipo de intervenciones } \\
\text { produjo mejores resultados. }\end{array}$ \\
\hline $\begin{array}{l}\text { Weston, Hodges y Davis } \\
\text { (2018) }\end{array}$ & $\begin{array}{l}\text { Revisión cualitativa de } 45 \text { estudios. } \\
\text { Total participantes: } 72 \text {. } \\
\text { Edad: } 3 \text { - } 26 \text { años. }\end{array}$ & $\begin{array}{l}\text { Refuerzo de conductas alternativas a las } \\
\text { conductas problemáticas. Las conductas a } \\
\text { modificar más habituales fueron: } \\
\text { estereotipias motores y vocales, agresiones } \\
\text { y autoagresiones. }\end{array}$ & $\begin{array}{l}\text { Aunque varias de las intervenciones de la revisión produjeron resultados positivos } \\
\text { con respecto a los participantes, el análisis de los indicadores de las publicaciones } \\
\text { no proporciona evidencia suficiente para asegurar que este procedimiento es un } \\
\text { tratamiento basado en la evidencia para el comportamiento desafiante de los niños } \\
\text { con TEA. }\end{array}$ \\
\hline
\end{tabular}




\section{Discusión}

Tras el análisis de los 10 estudios de revisión cualitativa y metaanálisis, se alcanzaron las siguientes conclusiones:

Actualmente, uno de los aspectos que más interesan a los diferentes grupos de investigación que trabajan sobre el TEA es la constatación de qué tipo de intervenciones han evidenciado empíricamente su eficacia y cuáles no han sido capaces de evidenciar esta eficacia de manera robusta. Ocho de los diez estudios revisados en el presente trabajo se publicaron entre los años 2017 y 2018, lo que supone un número de estudios de revisión notablemente elevado. Probablemente este interés deriva del trabajo realizado por el Council of Exceptional Children, que en 2014 publicó una serie de indicadores con la intención de determinar si los resultados de un estudio pueden o no considerarse una práctica basada en la evidencia (Cook et al., 2014). De esta manera, se podría determinar qué intervenciones merecen recibir la suficiente atención y es conveniente aplicar de manera mayoritaria en la práctica educativa.

Concretamente se trata de 8 indicadores que evalúan cuestiones que habitualmente sí suelen cumplirse en prácticamente todos los estudios publicados en revistas científicas, como la descripción detallada de los participantes, el contexto o el procedimiento de intervención. Pero que también incluyen otros indicadores que los estudios publicados cumplen en menor medida. Por ejemplo, uno de estos indicadores es la articulación de procedimientos para asegurar la fidelidad de las intervenciones, algo que no siempre ocurre cuando quien implementa la intervención es un agente diferente al propio investigador, como en el caso de las familias o el profesorado. Relacionado con ello, otro de los indicadores que se muestra crítico en numerosas investigaciones es asegurar la validez interna de la investigación. Habitualmente, las intervenciones con niños con TEA se llevan a cabo en contextos educativos, o en el contexto del hogar, donde es complicado controlar todas las variables que intervienen en los resultados finales, por lo que no siempre es posible afirmar con rotundidad que los resultados positivos obtenidos sean debidos únicamente a la intervención (ya que las mejoras en los niños podrían achacarse, al menos en parte, a variables no controladas en las investigaciones).

Teniendo en cuenta estos indicadores, la segunda conclusión del presente trabajo es que, aunque la mayoría de las intervenciones incluidas en las revisiones de la literatura y metaanálisis del presente trabajo parece que obtienen resultados positivos, o parcialmente positivos, no podemos afirmar tajantemente que se trate siempre de intervenciones eficaces a partir de la evidencia. Para ello, es necesario que los resultados sean robustos en diferentes estudios que cumplan con los estándares propuestos por el Council of Exceptional Children (Cook et al., 2014).

La tercera gran conclusión que se deriva de esta revisión es que las intervenciones basadas en análisis conductual aplicado sí parecen cumplir con estos estándares, además de ofrecer resultados positivos, aunque en diferente grado, en función del objetivo trabajado. El metaanálisis de Makrygianni et al. (2018), muestra que las intervenciones basadas en este procedimiento son altamente efectivas para mejorar variables como las habilidades intelectuales o de comunicación. Habitualmente, estas variables pueden ser evaluadas a través de tareas psicológicas que ofrecen puntuaciones estandarizadas, y que requieren habilidades similares a las que han sido entrenadas durante la intervención basada en análisis conductual aplicado. Se trata, por tanto, de tareas de contenido muy relevante para el TEA, pero que no siempre se pueden extrapolar a la vida diaria de los niños con TEA, por ser quizá excesivamente artificiales. 
De hecho, el metaanálisis de Makrygianni et al. (2018) indica que estas intervenciones obtienen resultados mucho menos positivos (los resultados caen en el rango de efectividad baja), cuando se evalúa su efectividad en habilidades de la vida diaria. Este tipo de habilidades están más desligadas de las tareas de evaluación estandarizadas, y hacen referencia a aspectos que podríamos considerar que son la extrapolación en el día a día de las habilidades que sí han mejorado gracias a la intervención. Por tanto, encontramos aquí una situación controvertida, ya que los resultados derivados de estas intervenciones sí podemos afirmar que son positivos y basados en la evidencia, pero estos resultados no consiguen realmente transferirse a la vida diaria de los niños con TEA, una situación similar a la que ocurre con las mejoras alcanzadas por las intervenciones encaminadas a trabajar con el lenguaje pragmático (Parsons et al., 2017).

Precisamente, el papel de las familias como agentes de intervención, podría ser una buena estrategia para intentar aumentar la transferencia de los resultados positivos a la vida diaria de los niños con TEA. Cuando los padres siguen estrategias educativas que les han proporcionado especialistas en el tema, lo hacen en contextos naturales, en el día a día de los niños, insertos en situaciones habituales en el ejercicio de la paternidad como en cualquier hogar, situaciones muy alejadas de contextos artificiales o de laboratorio.

A este respecto, dos de las revisiones incluidas en el presente trabajo han analizado la efectividad de las intervenciones implementadas por los padres (Gerow et al., 2018; Nevill et al., 2018). Sin embargo, los resultados de estas revisiones no son todo lo positivos que cabría esperar. En el metaanálisis de Nevill et al. (2018), las mejoras producidas tras la intervención de las familias tuvieron una significatividad muy baja, casi inapreciable en algunos ámbitos. Por su parte, el metaanálisis de Gerow et al. (2018), si bien sí reconoce mejoras en los resultados tras las intervenciones de las familias, subraya la ausencia de control de la fidelidad de la intervención, lo que no permite afirmar tajantemente que se trata de intervenciones eficaces y basadas en la evidencia. En cualquier caso, aunque los resultados no permiten hablar de resultados robustos, ambas revisiones afirman claramente que sí hay beneficios en la formación de las familias. Aunque estos beneficios no se vean claramente reflejados en los resultados de los metaanálisis, sí mejoran otros aspectos difíciles de controlar, como la comprensión y aceptación del diagnóstico y de las conductas y comportamientos de sus hijos, lo que a su vez puede repercutir en disminuir los niveles de estrés parental, una variable que se ha relacionado de manera robusta con la mejora de la sintomatología en los niños (Hayes y Watson, 2013).

Otra estrategia interesante para mejorar la transferencia a situaciones naturales de los aprendizajes de los niños con TEA es el uso de historias sociales. El metaanálisis de McGill et al. (2014), muestra que esta técnica de intervención es eficaz para mejorar algunos aspectos de la sintomatología TEA, especialmente las conductas verbales. Las mejoras que se producen gracias a las historias sociales son más sencillas de transferir a situaciones naturales, ya que las historias se construyen basándose precisamente en estas situaciones, por lo que es menos complicado para los niños utilizar los aprendizajes realizados en su vida diaria cuando este se realiza mediante una historia social que cuando se realiza a través de una situación más artificial.

Por otro lado, otra conclusión relevante del presente trabajo es que, en algunos ámbitos de intervención, es necesaria mucha más investigación. Por ejemplo, en la revisión de Hillman (2018) sobre terapia basada en el juego, únicamente incluye 4 estudios de intervención. Aunque los resultados de estas intervenciones sean prometedores, no podemos afirmar todavía que se trate de una intervención eficaz basada en la evidencia, sino que es necesario que estos resultados se confirmen en sucesivas intervenciones de manera consistente, antes de generalizar este tipo de 
intervenciones. Igualmente, en la revisión de Weston et al. (2018) sobre refuerzo de conductas alternativas a la conducta problemática, los resultados de los estudios revisados son positivos para alcanzar el objetivo propuesto. Sin embargo, es necesario continuar realizando investigaciones que cumplan con los estándares propuestos por el Council of Exceptional Children, antes de poder afirmar que se trata de intervenciones eficaces.

Finalmente, debemos remarcar también que las intervenciones revisadas en el presente trabajo no son ni mucho menos excluyentes. Por ejemplo, el análisis conductual aplicado y la metodología $\mathrm{TEACCH}$, ambos procedimientos que han mostrado su eficacia en diferentes estudios, comparten numerosos puntos en común, y pueden ser implementados conjuntamente en la escuela. Además, las intervenciones realizadas en el contexto escolar no deben ser excluyentes de las realizadas por la familia, sino que la situación ideal es que ambas intervenciones se realicen simultáneamente y de manera coordinada. De hecho, cuando se ha intentado comparar diferentes aproximaciones de intervención son más eficaces, ha sido difícil encontrar diferencias claras en los resultados de los diferentes procedimientos (Tachibana et al., 2017).

Consideramos que los resultados de este trabajo pueden ser relevantes para la práctica educativa, ya que proporcionan algunas certezas, y también algunas cuestiones para la reflexión sobre la eficacia de las intervenciones en niños con TEA. Estas cuestiones pueden servir de guía para los profesionales de la educación, ya que están basadas en el esfuerzo de investigadores de todo el mundo preocupados por la mejora de las estrategias de intervención educativa de los niños con TEA. Pero hay muchos otros aspectos, no sencillos de evaluar ni calibrar, que también tienen una gran relevancia en la educación de los niños con TEA. Se trata de aspectos como la vocación educativa de los profesionales, la capacidad de crear un buen clima de aula, de ejercer de líder de los procesos de inclusión educativa en un centro, del compromiso profesional con la realización de un trabajo de calidad, con la capacidad de colaborar con otros profesionales, y también de empatizar y conectar con los niños con TEA y sus familias. Estos factores, aunque complicados de medir en un estudio experimental, son también requisitos indispensables para la práctica educativa, y sin su presencia es muy difícil que las intervenciones educativas alcancen los resultados deseados, incluso aunque sigan fielmente los procedimientos descritos en los mejores artículos científicos.

\section{Referencias}

Los artículos incluidos en la revisión están marcados con asterisco*:

Cook, B., Buysse, V., Klingner, J., Landrum, T., McWilliam, R., Tankersley, M., \& Test, D. (2014). Council for Exceptional Children: Standards for evidence-based practices in special education. Teaching Exceptional Children, 46(6), 206.

Fortea, MS., Escandell, MO., y Castro, J. J. (2013). Estimación de la prevalencia de los trastornos del espectro autista en Canarias. Anales de Pediatría, 79(6), 352-359.

*Gerow, S., Hagan-Burke, S., Rispoli, M., Gregori, E., Mason, R., y Ninci, J. (2018). A systematic review of parent-implemented functional communication training for children with ASD. Behavior modification, 42(3), 335-363.
Hayes, SA., \& Watson, SL. (2013). The impact of parenting stress: A meta-analysis of studies comparing the experience of parenting stress in parents of children with and without autism spectrum disorder. Journal of Autism and Developmental Disorders, 43(3), 629-642.

*Hillman, H. (2018). Child-Centered Play Therapy as an Intervention for Children With Autism: A Literature Review. International Journal of Play Therapy, 27(4), 198-204.

Humphrey, N., y Hebron, J. (2015). Bullying of children and adolescents with autism spectrum conditions: A 'state of the field' review. International Journal of Inclusive Education, 19(8), 845-862.

*Makrygianni, M. K., Gena, A., Katoudi, S., y Galanis, P. (2018). The effectiveness of applied behavior analytic interventions for children with Autism Spectrum Disorder: A meta-analytic study. Research in Autism Spectrum Disorders, 51, 18-31. 
*McGill, RJ., Baker, D., \& Busse, R. T. (2015). Social Story ${ }^{\mathrm{TM}}$ interventions for decreasing challenging behaviours: a singlecase meta-analysis 1995-2012. Educational Psychology in Practice, 31(1), 21-42.

*Murza, K. A., Schwartz, J. B., Hahs-Vaughn, D. L., y Nye, C. (2016). Joint attention interventions for children with autism spectrum disorder: a systematic review and meta-analysis. International Journal of Language \& Communication Disorders, 51(3), 236-251.

*Nevill, R. E., Lecavalier, L., y Stratis, E. A. (2018). Meta-analysis of parent-mediated interventions for young children with autism spectrum disorder. Autism, 22(2), 84-98.

*Parsons, L., Cordier, R., Munro, N., Joosten, A., y Speyer, R. (2017). A systematic review of pragmatic language interventions for children with autism spectrum disorder. PloS One, 12(4), e0172242.

Russell, G., Collishaw, S., Golding, J., Kelly, S. E., y Ford, T. (2015). Changes in diagnosis rates and behavioural traits of autism spectrum disorder over time. British Journal of Psychiatry Open, 1(2), 110-115.
Sanz-Cervera, P., Fernández-Andrés, M. I., Pastor-Cerezuela, G., y Tárraga-Mínguez, R. (2017). Pre-Service Teachers' Knowledge, Misconceptions and Gaps About Autism Spectrum Disorder. Teacher Education and Special Education, 40(3), 212-224.

*Sanz-Cervera, P., Fernández-Andrés, M. I., Pastor-Cerezuela, G., y Tárraga-Mínguez, R. (2018). Efectividad de las intervenciones basadas en metodología TEACCH en el trastorno del espectro autista: un estudio de revisión. Papeles del Psicólogo, 39(1), 40-50

*Tachibana, Y., Miyazaki, C., Ota, E., Mori, R., Hwang, Y., Kobayashi, E., ... y Kamio, Y. (2017). A systematic review and meta-analysis of comprehensive interventions for pre-school children with autism spectrum disorder (ASD). PloS one, 12(12), e0186502

Vélez-Calvo, X., Tárraga-Mínguez, R., Fernández-Andrés, M. I., y Sanz-Cervera, P. (2016). Formación inicial de maestros en Educación Inclusiva: una comparación entre Ecuador y España. Revista de Educación Inclusiva, 9(3), 75-94.

*Weston, R., Hodges, A., y Davis, T. N. (2018). Differential Reinforcement of Other Behaviors to Treat Challenging Behaviors Among Children With Autism: A Systematic and Quality Review. Behavior Modification, 0145445517743487. 